\title{
AN IMBEDDING THEOREM FOR METRIC SPACES
}

\author{
STEPHEN LEON LIPSCOMB
}

\begin{abstract}
A simple solution to the imbedding problem for the class of separable metric spaces has been known for a long time: (URYSOHN's IMBEDDING THEOREM) A topological space is separable metric if, and only if, it can be imbedded in the topological product of countably many unit intervals. We see that products of the unit interval make an especially informative type of imbedding space since the finite (Lebesgue) dimensional separable metric spaces are those that can be imbedded in a finite product of intervals. The author has recently shown that this result concerning the finite case could be extended to arbitrary metric spaces if we use a topological generalization of the unit interval. This present paper shows that if we use this same generalization of the interval, then we can obtain an analogue to Urysohn's Imbedding Theorem. Besides presenting the first unified results which simultaneously generalize both separable cases, this paper contains comparisons with existing imbedding theorems and imbedding spaces.
\end{abstract}

1. Introduction. In this paper we establish an imbedding theorem for metric spaces (Theorem 2). This theorem represents the "limiting case" of an imbedding theorem (Theorem 1) for metric spaces of finite dimension. (The dimension concept is the Lebesgue or covering dimension.)

Before either of these theorems can be understood, we must give some definitions and make a few explanations: Let $\tau$ be an infinite cardinal and let $A$ be a set of cardinality $\tau$, i.e., $\aleph(A)=\tau$. Let $N(A)$ be Baire's zerodimensional space. $(N(A)$ is topologically a product space having a countable infinity of factor spaces-each homeomorphic to a discrete space of $\boldsymbol{\kappa}(A)$.) Two points $\left(\alpha_{1}, \alpha_{2}, \ldots\right),\left(\beta_{1}, \beta_{2}, \ldots\right) \in N(A)$ are $R$-related if, and only if, $\alpha_{i}=\beta_{i}$ for all $i \geqslant 1$, or, in case there exists a $j$ such that (1) $\alpha_{i}=\beta_{i}$ for $i<j$, (2) $\alpha_{j} \neq \beta_{j}$, and (3) $\alpha_{j}=\beta_{j+i}$ for all $i \geqslant 1$ and $\beta_{j}=\alpha_{j+i}$ for all $i \geqslant 1$. Let $N(A) / R=J(\tau)$ where $J(\tau)$ is given the quotient topology induced by the natural map $\varphi: N(A) \rightarrow N(A) / R$, i.e., $R$ is an equivalence relation. Then $z \in J(\tau)$ is rational if $\aleph\left(\varphi^{-1}(z)\right)=2$, and $z$ is irrational if $\aleph\left(\varphi^{-1}(z)\right)=1$. This is a topological generalization of identifying adjacent end points in the Cantor Space. Indeed, a copy of unit interval, together with subspaces of rational and irrational points, can be obtained by using these definitions. It is only required that one use the Cantor Space considered as the countable product of a two point discrete space instead of $N(A)$.

With this terminology we can state both the imbedding theorem for metric

Presented to the Society, January 31, 1975; received by the editors February 2, 1975.

AMS (MOS) subject classifications (1970). Primary 54E35, 54B15; Secondary 54F45, 54C25.

Key words and phrases. Imbedding metric spaces, metrizability, nonseparable metric spaces, covering dimension and imbedding. 
spaces of finite dimension (see [6]) and the new imbedding theorem for general metric spaces.

THEOREM 1. A topological space of weight $\tau \geqslant \kappa_{0}$ is metric of dimension $\leqslant n$ if, and only if, it can be imbedded in the set of points of $J(\tau)^{n+1}$ which have at most $n$ rational coordinates.

Letting $n$ go to infinity we get the statement of an imbedding theorem for metric spaces. The proof of this theorem is given in the following sections.

Theorem 2. A topological space of weight $\tau \geqslant \kappa_{0}$ is metric if, and only if, it can be imbedded in $J(\tau)^{\infty}$, i.e., in the product of countably many copies of $J(\tau)$.

Before moving on to a discussion and proof of Theorem 2 one important comment concerning Theorem 1 should be made: The author has learned from R. Engelking that K. Borsuk recently proved that the two-sphere cannot be imbedded in a product of two one-dimensional spaces. Since $J(\tau)$ is one dimensional, we see that Theorem 1 is the best that can be obtained for the finite case when we consider only finite products of the space $J(\tau)$.

2. Discussion. This pair of theorems should be compared to the corresponding pair of classical theorems for the separable metric case. (Theorems 3 and 4 below; see [2, p. 64] and [3, p. 125].) In view of the definition of $J(\tau)$ above, the desirable parallel between the general pair and the separable pair of theorems is clear.

Theorem 3 (Classical Imbedding Theorem; see [10]). A topological space of weight $\tau=\kappa_{0}$ is metric of dimension $\leqslant n$ if, and only if, it can be imbedded in the set of points of $I^{2 n+1}$ which have at most $n$ rational coordinates. $\left(I^{2 n+1}\right.$ denotes the product space of $2 n+1$ copies of the unit interval I.)

Theorem 4 (Urysohn's ImbedDing TheOrem). A topological space of weight $\tau=\aleph_{0}$ is metric if, and only if, it can be imbedded in $I^{\infty}$, i.e., in the product space of countably many copies of the unit interval $I$.

Further, Theorems 1 and 2 should be contrasted with the corresponding general imbedding theorems due to J. Nagata [7] and H. J. Kowalsky [4]. (Theorems 5 and 6 below; see [8, p. 184] and [9, p. 207].) For this, however, we will need the definition of a star-space with index $A$ : Let $\{I(\alpha) \mid \alpha \in A\}$ be a system of unit segments $[0,1]$. By identifying all zeros in $\cup\{I(\alpha) \mid \alpha \in A\}$ we get a star-shaped set $S(A)$. Defining a metric $d$ in $S(A)$ by

$$
d(x, y)=\left\{\begin{array}{cl}
|x-y| & \text { if } x, y \text { belong to the same segment } I(\alpha), \\
x+y & \text { if } x, y \text { belong to distinct segments }
\end{array}\right.
$$

we obtain a metric space called star-shaped with index $A$. Let $S(A)^{\infty}$ denote the product of countably many spaces $S(A)$.

Theorem 5 (NagAta). Let $\boldsymbol{\aleph}(A)=\tau$. A topological space of weight $\tau \geqslant \aleph_{0}$ is metric of dimension $\leqslant n$ if, and only if, it can be imbedded in the set of points of $S(A)^{\infty}$ at most $n$ of whose nonvanishing coordinates are rational.

Theorem 6 (Kowalsky). Let $\mathbf{N}(A)=\tau$. A topological space of weight 
$\tau \geqslant \aleph_{0}$ is metrizable if, and only if, it is homeomorphic with a subspace of $S(A)^{\infty}$.

We note that the statement of Theorem 6 is in some sense the limit of the statement of Theorem 5 , as $n$ goes to infinity. However, this latter "limit process" is not the same as the former, i.e., in the latter case we have an infinite product space in each finite statement while in the two former cases we had a finite product space for each finite statement.

Also, just as Theorem 1 is the best of its type, we can see that Nagata's theorem is the best theorem obtainable under the assumption that one must use star-spaces and form product spaces. This follows since any product of finitely many star-spaces is a metric space with a $\sigma$-star-finite open base while not every finite dimensional metric space has a $\sigma$-star-finite open base as pointed out by $\mathrm{Yu}$. Smirnov.

It is shown below that if $\tau=\boldsymbol{\aleph}(A)$, then $S(A)$ is topologically imbeddable in $J(\tau)$. As a matter of fact a subset $F$ of $N(A)$ is chosen and mapped onto $S(A)$ in such a manner that the mapping involved is merely the relation $R$ restricted to $F \subset N(A)$. Since $S(A)$ could be defined in this way we see from previous remarks that all of the imbedding spaces $I, S(A)$, and $J(\tau)$ are unified under this one type of construction.

3. On the proof of Theorem 2. The key to proving Theorem 2 in this paper is an application of Theorem 6. We will show that if $\tau=\aleph(A)$, then $S(A)$ can be imbedded in $J(\tau)$. It then follows that $S(A)^{\infty}$ is topologically imbeddable in $J(\tau)^{\infty}$. With this then we can apply the necessary part of Theorem 6 to deduce the necessary part of Theorem 2. The sufficiency part of Theorem 2 follows easily from the fact that a product of countably many metric spaces is metric (to see a proof that $J(\tau)$ is metric see [5]). Thus, with these remarks, it only remains to see that $S(A)$ is topologically contained in $J(\tau)$. This is accomplished in the next section.

It is an open problem to obtain a proof of Theorem 2 using the techniques developed in [6].

4. $S(A)$ can be imbedded in $J(\tau)$. We will use the following theorem for the imbedding of $S(A)$ into $J(\tau)$. This theorem is standard, e.g., see [1, p. 123] of Dugundji's text.

THEOREM 7. Let $p: F \rightarrow X$ be a continuous onto quotient map and $f: F \rightarrow S$ be continuous. Assume that $f p^{-1}$ is single valued (that is, $f$ is constant on each fiber $p^{-1}(x)$ ). Then $f p^{-1}: X \rightarrow S$ is continuous. Also, $f p^{-1}$ is a a closed map if, and only if, $f(U)$ is closed whenever $U$ is a closed p-inverse set $\left(U=p^{-1} p(U)\right)$.

In order to apply Theorem 7 we will need the following: Let $\boldsymbol{\alpha}=\left(\alpha_{1}\right.$, $\left.\alpha_{2}, \ldots\right) \in N(A)$ be such that $\left\{\alpha_{i} \mid i=1,2 \ldots\right\}=C(\boldsymbol{\alpha}) \subset A$ is finite. In this case we say $\boldsymbol{\alpha}$ is of finite character. Also, we will call the members of $C(\boldsymbol{\alpha})$ the characters of $\boldsymbol{\alpha}$.

Now let $\alpha^{0} \in A$ be fixed throughout this section and define $A^{\prime}=A$ $-\left\{\alpha^{0}\right\}$. Let $F$ be all the members of $N(A)$ which have at most two characters with the restriction that when a member of $F$ has exactly two characters then one of these characters is $\alpha^{0}$. Thus, if we pick $\alpha \in A^{\prime}$ and let $\Pi_{i}\left\{\alpha^{0}, \alpha\right\}_{i}=F(\alpha)$ denote the product of countably many copies of the 
two-point discrete space $\left\{\alpha^{0}, \alpha\right\}$, then we can easily show that $F=\cup\{F(\alpha) \mid$ $\left.\alpha \in A^{\prime}\right\}$. Let $\chi$ be the characteristic function of $A^{\prime}$ defined on $A$, i.e., $\chi(\alpha)=1$ if $\alpha \in A^{\prime}$ and $\chi(\alpha)=0$ if $\alpha=\alpha^{0}$. Let $f: F \rightarrow S\left(A^{\prime}\right)$ be defined as follows: If $\beta \in F(\alpha)$ for some $\alpha \in A^{\prime}$, let

$$
f(\boldsymbol{\beta})=\sum \chi\left(\beta_{i}\right) / 2^{i} \in I(\alpha)
$$

where $\Sigma \chi\left(\beta_{i}\right) / 2^{i}$ denotes the infinite series summed over the index $i \geqslant 1$. With these statements we can prove the following lemmas.

Lemma 1. Let $f: F \rightarrow S\left(A^{\prime}\right)$ be defined as above. Let $\boldsymbol{\eta} \in F$ and let $\left\{\xi^{j}\right\}$ be a sequence in $F$ which converges to $\boldsymbol{\eta}$ in $F$. Then the sequence $\left\{f\left(\xi^{j}\right)\right\}$ converges to $f(\boldsymbol{\eta})$ in $S\left(A^{\prime}\right)$.

Proof. In view of the topologies on $F$ and $S\left(A^{\prime}\right)$ it clearly suffices to show that for two points $\boldsymbol{\xi}, \boldsymbol{\eta} \in F$ such that $\xi_{i}=\eta_{i}$ for $i=1, \ldots, k$ we necessarily have $d(f(\xi), f(\boldsymbol{\eta})) \leqslant 1 / 2^{k-1}$. To show this we first assume that there is an $\alpha \in A$ such that $\xi, \eta \in F(\alpha)$. If $\xi_{i}=\eta_{i}$ for $i \leqslant k$, then it follows from the definition of $f$ and $d$ that $d(f(\xi), f(\boldsymbol{\eta})) \leqslant \Sigma 1 / 2^{i}$ (where this sum is over those $i>k)$. But this last sum is equal to $1 / 2^{k}$ which is less than $1 / 2^{k-1}$. For the second part we assume that there is no $\alpha \in A$ such that both $\xi$ and $\boldsymbol{\eta}$ belong to $F(\alpha)$. Then $\xi_{i}=\eta_{i}$ for $i \leqslant k$ implies $\xi_{i}=\alpha^{0}=\eta_{i}$ for $i \leqslant k$. Hence $\chi\left(\xi_{i}\right)=0=\chi\left(\eta_{i}\right)$ for $i \leqslant k$. Thus, from the definitions of $f$ and $d$ it follows that

$$
d(f(\xi), f(\boldsymbol{\eta})) \leqslant\left(1 / 2^{k}\right)+\left(1 / 2^{k}\right)=1 / 2^{k-1} .
$$

Therefore, from the first statement above this proof is complete.

Lemma 2. Let $M \subset S\left(A^{\prime}\right)$ be such that $0 \notin \operatorname{cl}(M)$. If $M \cap I(\alpha)$ is closed in $I(\alpha)$ for each $\alpha \in A^{\prime}$, then $M$ is closed in $S\left(A^{\prime}\right)$.

Proof. Suppose that $M$ is not closed in $S\left(A^{\prime}\right)$. Then there is an $x \in S\left(A^{\prime}\right)$ such that $x \in \operatorname{cl}(M)-M$. Now clearly $x \in I(\alpha)-\{0\}$ for some $\alpha \in A^{\prime}$. However, $I(\alpha)-\{0\}$ is an open subset of $S\left(A^{\prime}\right)$ and therefore $x$ is in the closure of $M \cap(I(\alpha)-\{0\})=M \cap I(\alpha)$. Since $M \cap I(\alpha)$ is closed in $I(\alpha)$ we can see that $x$ must be in $M$. Thus we have a contradiction and we are led to the conclusion that $M$ is closed in $S\left(A^{\prime}\right)$.

Lemma 3. Let $M \subset S\left(A^{\prime}\right)$ and $0 \in M$. If $M \cap I(\alpha)$ is closed in $I(\alpha)$ for each $\alpha \in A^{\prime}$, then $M$ is closed in $S\left(A^{\prime}\right)$.

Proof. The proof is similar to the proof of Lemma 2.

LEMMA 4. If $\boldsymbol{\alpha}^{0} \notin H$ where $H$ is a closed subspace of $F$, then $f\left(\boldsymbol{\alpha}^{0}\right)$ is not in the closure of $f(H)$, i.e., $f\left(\boldsymbol{\alpha}^{0}\right) \notin \operatorname{cl}(f(H))$.

Proof. If $H$ is closed and $\boldsymbol{\alpha}^{0} \notin H$ then there is an $m$ such that when $\boldsymbol{\beta} \in F$ and $\beta_{i}=\alpha^{0}$ for all $i \leqslant m$ then $\boldsymbol{\beta} \notin H$. It is easy to see that the set $\left\{\boldsymbol{\beta} \mid d\left(f\left(\boldsymbol{\alpha}^{0}\right), f(\boldsymbol{\beta})\right)<1 / 2^{m}\right\}$ is therefore disjoint from $\boldsymbol{H}$. The conclusion clearly follows.

TheOREM 8, The map $f: F \rightarrow S\left(A^{\prime}\right)$ is a closed map.

Proof. Let $H$ be a closed subset of $F$. There are two cases to consider: 
First, suppose $\boldsymbol{\alpha}^{0} \notin H$. Then since $H \cap F(\alpha)$ is compact and $f$ is continuous (this follows directly from Lemma 1) we see that $f(H \cap F(\alpha))=f(H) \cap$ $I(\alpha)$ is a compact subset of $I(\alpha)$ and, hence, closed in $I(\alpha)$ for each $\alpha \in A^{\prime}$. By Lemma $4, f\left(\boldsymbol{\alpha}^{0}\right) \notin \operatorname{cl}(f(H))$ and, hence, by Lemma 2 we see that $f(H)$ is closed in $S\left(A^{\prime}\right)$. This completes the case where $\boldsymbol{\alpha}^{0} \notin H$. Second, we suppose $\boldsymbol{\alpha}^{0} \in H$.In this case $f\left(\boldsymbol{\alpha}^{0}\right)=0 \in f(H)$ and a compactness argument similar to the one used in the first case, together with Lemma 3, can be used to show that $f(H)$ is closed in $S\left(A^{\prime}\right)$. This therefore completes this proof.

THEOREM 9. If $\boldsymbol{\aleph}(A)=\tau \geqslant \aleph_{0}$, then $S(A)$ can be imbedded in $J(\tau)$.

Proof. We will make an application of Theorem 7. To do this we will let (1) $F$ be the subspace of $N(A)$ that we defined above, (2) $X$ be the image of $F$ under the natural map $\varphi$ from $N(A)$ to $J(\tau)$, and (3) $S$ be the space $S\left(A^{\prime}\right)$ that we defined above. For maps, we will let $p$ be the natural map $\varphi$ restricted to $F \subset N(A)$, and we will let $f$ be the map from $F$ onto $S\left(A^{\prime}\right)$ that we defined above. With this notation we now show that the first sentence of Theorem 7 is true. The continuity of $p$ is clear while the continuity of $f$ follows easily from Lemma 1. To see that $p$ is also a quotient map we note firstly that $\varphi$ is a closed map (see [5]) and, consequently, $\varphi$ restricted to any $\varphi$-inverse set is a closed map. Secondly, we recall that a continuous closed map is quotient. Thus, since $F$ is a $\varphi$-inverse set and $p$ is $\varphi$ restricted to $F$ we see that $p$ is a quotient map. Therefore the first sentence of Theorem 7 holds. It is easy to see that the second sentence is also true. Thus $f p^{-1}: p(F) \rightarrow S\left(A^{\prime}\right)$ is continuous. To see that $f p^{-1}$ is a closed map we will use the last sentence of Theorem 7. To use this last sentence it suffices to show that $f$ is a closed map. But this is precisely the statement of Theorem 8 . Thus $f p^{-1}: p(F) \rightarrow S\left(A^{\prime}\right)$ is a continuous closed map. To see that $f p^{-1}$ is one-one and onto is easy. Thus $f p^{-1}$ is a homeomorphism and it only remains to observe that $S\left(A^{\prime}\right)$ is obviously homeomorphic to $S(A)$.

\section{BIBLIOGRAPHY}

1. J. Dugundji, Topology, Allyn and Bacon, Boston, Mass., 1966. MR 33 \# 1824.

2. W. Hurewicz and H. Wallman, Dimension theory, Princeton Math. Ser., vol. 4, Princeton Univ. Press, Princeton, N.J., 1941. MR 3, 312.

3. J. L. Kelley, General topology, Van Nostrand, Princeton, N.J., 1955. MR 16, 1136.

4. H.-J. Kowalsky, Einbettung metrischer Räume, Arch. Math. 8 (1957), 336-339. MR 19, 971.

5. S. L. Lipscomb, Imbedding one-dimensional metric spaces, Dissertation, University of Virginia, 1973.

6., On imbedding finite-dimensional metric spaces, Trans. Amer. Math. Soc. 211 (1975), 143-160.

7. J. Nagata, A remark on general imbedding theorems in dimension theory, Proc. Japan Acad. 39 (1963), 197-199. MR 29 \# 1616.

8. , Modern dimension theory, Bibliotheca Math., vol. 6, Interscience, New York, 1965. MR 34 \# 8380 .

9. ___ Modern general topology, Bibliotheca Math., vol. 7, North-Holland, Amsterdam; Interscience, New York, 1968. MR 41 \#9171.

10. G. Nöbeling, Über eine $n$-dimensionale Universalmenge in $R_{2 n+1}$, Math. Ann. 104 (1930), 71-80.

DK-55 Naval Surface Weapons Center, Dahlgren, Virginia 22448 